\title{
TRACHOMA AS ONE OF THE LEADING CAUSE OF LOW VISION IN KANO STATE
}

\author{
BY \\ *ODJIMOGHO, E. S. AND ODJIMOGHO, S. E. \\ DEPARTMENT OF OPTOMETRY, UNIVERSITY OF BENIN, \\ BENIN-CITY, NIGERIA. \\ Email: stellare@yahoo.com \\ *Corresponding author
}

\begin{abstract}
Trachoma is fast becoming a common cause of low vision in developing countries. In order to ascertain this, a survey of one thousand (1000) subjects with low vision aged between 15-70 years with mean age of 31.30 years was conducted in the northern part of Nigeria. These subjects were seen from 1990-1998 (a period of 9 years) in three leading specialist eye centres in Kano State (ECWA Savana and General hospital). Data on sex, age, cause of low vision, and the visual acuity was obtained and analysed using percentages. The result revealed that, trachoma was the second highest leading cause of blindness after cataract with 243 subjects representing (24.3\%) of the sample population. And female subjects were more affected than their male counterpart with $69.1 \%$ and $30.9 \%$ respectively. The most affected age group was 5-15 years with 50 (20.6\%) of the sample population and the least affected age group was 60-70 years with 30 representing (12.3\%). Also $85.5 \%$ of the study population had visual acuity of 20/1000 while $14.5 \%$ had acuity of 20/700. And all the subject in this study population had the infection at terminal stage. In conclusion therefore, the study has shown that trachoma is the second highest cause of partial blindness or low vision, with more than $20 \%$ of the sample population affected. Thus there is need for urgent enlightenment on preventive measure to save our citizen especially those in Northern Nigeria from the menace of trachoma.
\end{abstract}

KEYWORDS: Trachoma, Cataract, Low vision, Chlamydia trachomatis.

\section{INTRODUCTION}

Low vision is a state of visual impairment that would result in the inability of the individual to perform normal visual task with regular spectacle. It is a form of visual handicap characterized by a subnormal vision of $<20 / 200$ or constricted visual field defect of $<20$ degrees in the better eye. One major cause of this condition especially in the Northern part of Nigeria is trachoma.

Trachoma is a chronic contagious Chlamydia infection of the conjunctiva, cornea and the eyelids. The causative micro-organism, Chlamydia trachomatis, has some traits of a virus, behaves like a bacterium, though multiplies intracellularly, and of different stereotype A, B, Ba and C. It is associated with ignorance, poor economic status, personal hygiene and health care. It is also endemic in dry, hot and dusty climatic environment and could be seasonal ${ }^{1}$.

Trachoma infection starts with the attachment of elementary bodies to the surface of the conjunctiva epithelium. This body further divides metabolically by binary fission to produce cluster of particles, which are bond together in glycogen matrix after 18-24 hours of infection and can only be observed with Giemsa stain. The process of infection is in 4 stages namely;-The incipient stage (1), follicular hypertrophy stage (II), cicatrizing trachoma stage (III), and healed trachoma (IV).

The stage $\mathrm{I}$ is characterized by matured follicles on the superior tarsus with minimal hypertrophy. Early corneal changes such as punctuate keratitis or a fine superior panus may be present. Stage II is classified into two namely: (A) follicular hypertrophy which is characterized by mature follicles in the superior tarsus that can rapture under pressure, limbal follicles, pain, corneal sub-epithelial infiltrates extending from the lower limbus. And stage (B) papillary hypertrophy, which is characterized by intensification of the inflammatory response along with obliteration of the follicles papillary hypertrophy accompanied with pains, superior paralimbal corneal infiltrates and necrosis of the follicles. Stage III (cicatrizing trachoma), characterized by scarring necrotic follicles, and panus is often grossly visible. Limbal follicles begin to cicatrize and form pit (Herbet's pits). Fine lined scar may appear in the tarsal conjunctiva (Arlt's lined), entropion and trichiasis with 
conjuntival cicatrization (i. e. conjunctiva and lid deformation). While stage IV (healed trachoma) is characterized by conjunctiva scar from resolved inflammation, entropion, trichiasis, dry eye, corneal erosion and blind complication ${ }^{2,3}$.

In endemic area, it affects virtually all children between the ages of 1-2 years and usually spread within families by direct contact with infected person or by the common housefly. The prevalence decreases with age and in prolong cases it can be exacerbated by bacteria infections. It is generally characterized by follicular conjunctivitis 15 days after inoculation and in acute state it is almost bilateral and associated with tearing, hyperemia, photophobia and mucopurulent discharge. It is the second most common cause of blindness after cataract and it is treated with sulphonamide ${ }^{1}$.

The World Health Organisation estimated a few years ago that about 500million people are affected by trachoma, 200 million visually handicapped (low vision) and up to 79 million completely blind from the disease ${ }^{4}$. The prevalence of this disease, as a common cause of low vision has been reported by researchers in some African countries (Saudi Arabia, Tanzania, Sudan, Nigeria $)^{5,6,7,}$, reports also have it that this disease affects more of the younger age group than the older ones. This has been corroborated in the Community eye health publication, which stated that there is currently an estimated 140 million children with active trachoma that can lead to low vision if the situation is not prevented by strict observation of proper hygiene as well as result of study conducted by Aouchichie ${ }^{9,10}$. Some other reports also have that there is an estimated 10 million people (mainly women) who are at risk of getting blind from trachoma. This is corroborated by study conducted by Munoz's ${ }^{11}$ whose result revealed that females are more frequently affected by the disease than the males ${ }^{11 .}$

\section{MATERIALS AND METHOD}

A survey of 1000 low vision subjects aged between 5-70 years with mean age $33.36 \pm 17.75$ seen from 1990-1998 (a period of nine years) in three major hospitals (ECWA, Savana, General Hospital), in Kano State was conducted. The cause of low vision was identified. Only those whose condition was caused by trachoma were selected for the study population. Data on sex, age, stage of the disease, visual acuity were obtained from the records. The stage of the disease was assessed with the slit-lamp biomicroscope, while the visual acuity was taken with the Sloan's acuity charts for both distance and near. Those with trachoma were analysed using percentages to ascertain its prevalence in the study population as well as determine if there is any age and gender relationship.

\section{RESULT}

Table 1 showed a percentage distribution of the causes of low vision in the study population. The result showed that trachoma is the second highest cause of low vision with 243 subjects representing $24.3 \%$ of the study population.

Table 2 showed the frequency distribution of those with trachoma in the 3 major hospitals. ECWA had the highest with 123 subjects representing $50.62 \%$ of the study population, followed by General Hospital with 73(30.04\%) and Savana with 47(19.34) of the study population.

Table 3 and fig. 1 showed the age and sex distribution of the disease in the study population. The most affected age group is 5-15 years with 50 representing $20.6 \%$ of the sample population and the least affected age group was $60-70$ years with 30 representing $12.3 \%$ of the study population. 208 subjects representing $85.5 \%$ of the study population had VA $<20 / 200$ while $35(14.5 \%)$ had VA of $<20 / 700$. All subjects in the study population had their disease in the terminal stage (IV).

\section{DISCUSSION}

Table I showed that Trachoma affects more than $24 \%$ of the study population making it the second highest cause of low vision in the sample population. This conforms to the World Health Organization (WHO) report that the disease may affect an estimated $20 \%$ of the world's population. The high prevalence of the disease in the study population is in line with studies conducted by some researchers ${ }^{4.8}$ who reported high prevalence of the disease in their study. The reason for the high prevalence in the population may be due to the dry, dusty and hot climatic nature of Kano state, which favours the easy spread of the disease at specific period of the year.

Table 2 showed that of the total study population of 1000, $243(24.3 \%)$ had trachoma. A breakdown of this number showed that 75 $(30.86 \%)$ were males while $168(69.14 \%)$ were females. This gives the proportion of male and female afflicted by the disease in the study population approximately in the ratio of $1: 2$.

Table 3 showed that the most affected age group is 5-15years with $50(20.6 \%)$ of the sample population followed by 27-37 years with 
$48(19.8 \%)$ and least was $60-70$ years with 30 $(12.3 \%)$. This corroborated with studies conducted by some researchers ${ }^{9,10}$ which revealed that the younger age group is more affected than the older age group. Reason being that children constitute a larger proportion of the population and have lower resistance to attacks from harmful microorganisms, hence, are more prone to infection than adults. Also because the spread of this disease occurs more frequently by physical contact with infected person, non-observance of good personal hygiene and overcrowding, children are mainly the victims. Table 3 also showed that females were more affected in the sample population than males with $168(69.1 \%)$ and 75 $(30.9 \%)$ respectively. This is in line with results obtained by some researchers ${ }^{9,10}$, who reported that women accounts for $2 / 3$ of those with blindness from trachoma. This might be due to the fact that most women especially those in the rural communities are of low economic status, low literacy level and poor hygiene maintenance culture. All of these are risk factors, which would prepare the ground for the infection and the spread of this disease.

The study showed a high prevalence of trachoma in a dry, hot, and dusty environment such as Kano State. Its gender preference (affecting more females than males) as well as its affectation of the younger age group (5-15 years) was also established. Thus a proper enlightenment campaign to educate mothers on the need for the maintenance of a good personal and environmental hygiene, as well as taking proper care of the children, should be embarked upon in other to eliminate or drastically reduce the rate of the infection and spread of the disease. This will help to minimize the prevalence of trachoma as one of the avoidable causes of blindness in our environment since it is the responsibility of the mothers to cater for the children.

\section{TABLE 1: FREQUENCY DISTRIBUTION OF THE CAUSES OF LOW VISION ON} THE STUDY POPULATION

\begin{tabular}{|l|l|l|}
\hline Causes of low vision & Frequency & \% \\
\hline Cataract & 305 & 30.5 \\
\hline Trachoma & 243 & 24.3 \\
\hline Glaucoma & 128 & 12.8 \\
\hline Onchocerciasis & 85 & 8.5 \\
\hline Xeropthamia & 60 & 6 \\
\hline Macular Degeneration & 37 & 3.7 \\
\hline Diabetic Retinopathy & 34 & 3.4 \\
\hline Unidentified cause & 108 & 10.8 \\
\hline Total & 1000 & 100 \\
\hline
\end{tabular}

TABLE 2: AGE DISTRIBUTION OF SUBJECT WITH TRA CHOMA IN RELATION TO GENDER IN SAMPLE POPULATION

\begin{tabular}{|l|l|l|l|l|l|l|}
\hline $\begin{array}{l}\text { Age Range in } \\
\text { years }\end{array}$ & Male & $\mathbf{\%}$ & Female & $\mathbf{\%}$ & Total & $\%$ \\
\hline $5-15$ & 14 & 5.8 & 36 & 14.8 & 50 & 20.6 \\
\hline $16-26$ & 16 & 6.6 & 31 & 12.8 & 47 & 19.4 \\
\hline $27-37$ & 15 & 6.8 & 33 & 13.6 & 48 & 19.8 \\
\hline $38-48$ & 12 & 4.9 & 21 & 8.6 & 33 & 13.6 \\
\hline $49-59$ & 9 & 3.7 & 26 & 10.7 & 35 & 14.4 \\
\hline $60-70$ & 9 & 3.7 & 21 & 8.7 & 30 & 12.3 \\
\hline Total & 75 & $(30.9)$ & 168 & 69.1 & 243 & 100 \\
\hline
\end{tabular}


TABLE 3: AGE DISTRIBUTION OF POPULATION WITH TRACHOMA IN THE THREE DIFFERENT SPECIALIST EYE CENTRES.

\begin{tabular}{|l|l|l|l|l|l|l|l|l|l|l|}
\hline \multicolumn{3}{|l|}{} & ECWA & \multicolumn{3}{l|}{ Savana } & \multicolumn{3}{|l|}{} & \multicolumn{3}{l|}{ General Hospital } & Total \\
\hline $\begin{array}{l}\text { Age } \\
\text { range }\end{array}$ & $\mathbf{M}$ & $\mathbf{F}$ & T & $\mathbf{M}$ & $\mathbf{F}$ & $\mathbf{T}$ & $\mathbf{M}$ & $\mathbf{F}$ & $\mathbf{T}$ & T \\
\hline $5-15$ & 8 & 21 & 29 & 2 & 6 & 8 & 4 & 9 & 13 & 50 \\
\hline $16-26$ & 7 & 17 & 24 & 3 & 5 & 8 & 6 & 9 & 15 & 47 \\
\hline $27-37$ & 9 & 22 & 31 & 2 & 3 & 5 & 4 & 8 & 12 & 48 \\
\hline $38-48$ & 5 & 7 & 12 & 3 & 4 & 7 & 4 & 10 & 14 & 33 \\
\hline $49-59$ & 4 & 15 & 19 & 3 & 6 & 9 & 2 & 5 & 7 & 35 \\
\hline $60-70$ & 2 & 6 & 8 & 3 & 7 & 10 & 4 & 8 & 12 & 30 \\
\hline Total & 35 & 88 & $123,50.62 \%$ & 16 & 31 & $47,19.34 \%$ & 24 & 49 & $73,30.04 \%$ & 243 \\
\hline
\end{tabular}

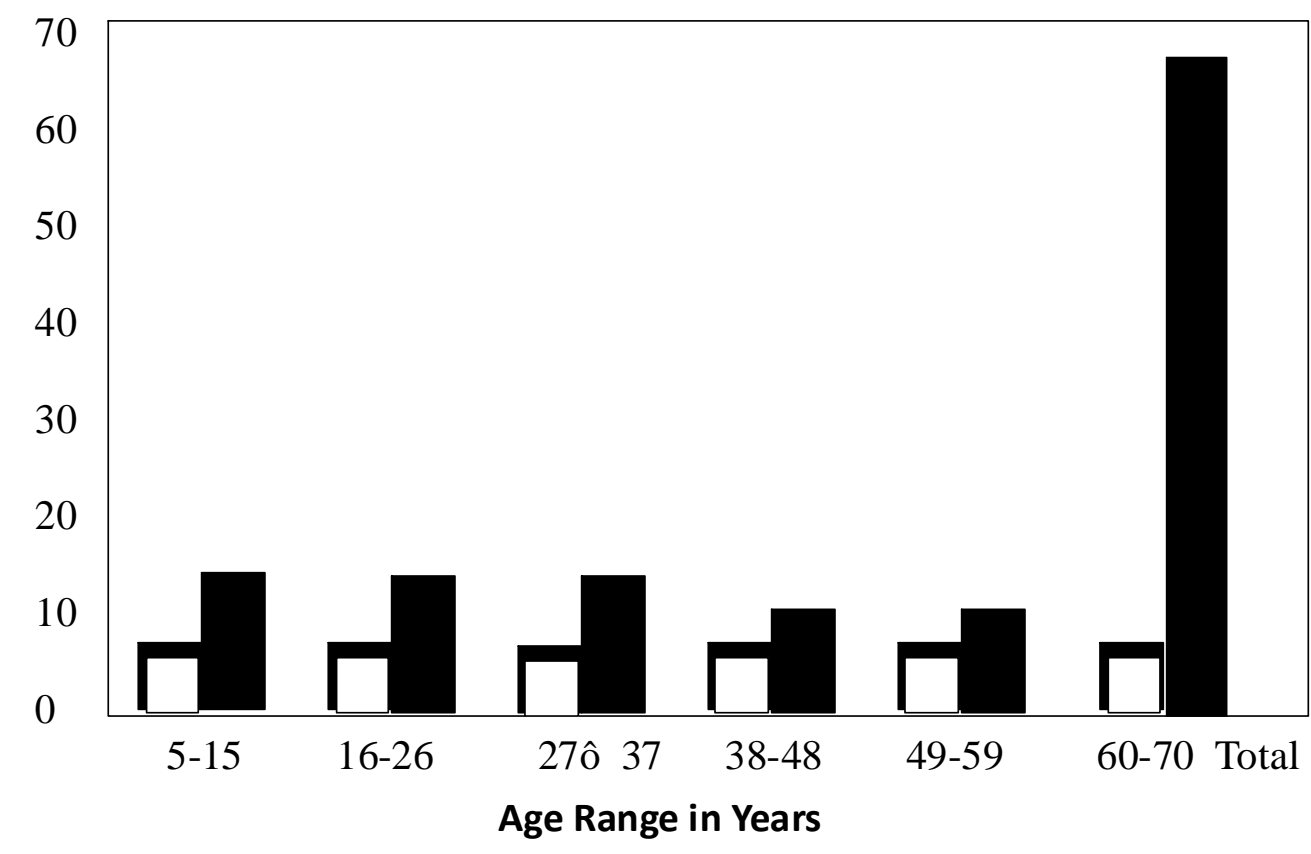

Fig. 1: AGE AND SEX DISTRIBUTION OF TRACHOMA

\section{R E F E R E N C E S}

1. Eleanor, E. and Faye, M. D. (1998): Chemical II: Low vision, pp 4-9.

2. Voughan D. A. (1980): General Ophthalmology. CALange, Los Altos, pp68-9.

3. Wang, L. H. (1992): Application of the new trachoma grading system. Chung, 28 (5): 2735.

4. Taylor, H. R., Millan valaslco, F., and Sommer, A. (1985): The ecology of Trachoma in Southern Mexico. WHO, 63:559-67.

5. Mahouts, E. A., and Sheikh, A. H. (1994): Children prevalence of Trachoma among displaced persons in Sudan. 8 (pt1): 130-3.

6. Ranson, M. K. and Evans, T. G. (1995): The global burden of trachomatous visual impairment: Assessing prevalence. Ophthalmol, 19(5): 261-70.

7. Obiliki, A. G. (1990): Prevalence of trachoma among school children in Plateau State. J. Ophthalmol, 67: 181-92.

8. Olurin, O. (1973): Causes of blindness in Nigeria: A case study of hospital patients. West Afri. Med.J, 23(6):97-102.

9. Community Eye Health (1998), II(26):18.19.

10. Aouchichie, M. (1987): Treatment and prevention of major causes of blindness in the world. Trop. Ocular Pathol. Publication (64):89-93.

11. Munoz, B., Aron, J. and Turner, V. (1997):

Tropical Medicine Int. Health. 